Universidad de Guadalajara

Derecho Global. Estudios sobre Derecho y Justicia

Año 3, núm 9, julio-octubre, 2018, pp. 131-154, ISSN: 2448-5128 e-ISSN: 2448-5136

https://doi.org/10.32870/dgedj.v0i9.169

\title{
Derecho y argumentación desde una perspectiva kelseniana
}

\section{Law and argumentation from a kelsenian perspective}

\author{
Alejandro César Antonio Luna Bernal
}

Miembro del Sistema Nacional de Investigadores, México.

Recibido: 02/03/2018

aluna642@hotmail.com

Aceptado: 15/04/2018

RESUMEN: El presente trabajo tiene como objetivo mostrar que dentro de una concepción kelseniana del sistema jurídico la argumentación resultaría ser una actividad imprescindible en el proceso de creación/aplicación del derecho. Se parte de la premisa interpretativa de considerar al orden jurídico como un sistema no puramente dinámico y, con base en ello, se realiza un análisis del pensamiento de Kelsen relativo al proceso de toma de decisiones judiciales con el fin de identificar contextos en los que tendría lugar la argumentación. Los hallazgos muestran que la teoría de Kelsen brinda un marco para conceptualizar cinco tipos de argumentación: a) fundamentadora, b) en materia de hechos, c) interpretativa, d) deliberativa, y, e) justificatoria. Con lo anterior, el trabajo contribuye a la discusión sobre las posibles aportaciones del pensamiento de Kelsen a la teoría contemporánea de la argumentación jurídica.

PALABRAS CLAVE: Argumentación jurídica, Democracia, Relativismo moral, Ciencia jurídica, Corrección racional.

ABSTRACT: The aim of this paper is to show that the argumentation is an essential activity in the process of creation/application of law within a Kelsenian conception of the legal system. The starting point is an interpretative premise: considering the legal order as a nonpurely dynamic system. Based on this, an analysis of Kelsen's thought regarding the judicial decision-making process is carried out in order to identify contexts in which the argumentation occurs. The findings show that Kelsen's theory provides a framework to conceptualize five types of argumentation in the judicial decision making: a) on validity of rules, b) on facts, c) interpretative, d) deliberative and e) justificatory. With all the above, this research feeds the discussion about the possible contributions of Kelsen's thought to the contemporary theory of legal argumentation.

KEY WORDS: Legal argumentation, Democracy, Moral relativism, Legal science, Rational correction. 


\section{SUMARIO}

I. Introduccion. II. Fundamentacion y argumentación. III. El sistema jurídico no es puramente dinámico. IV. Función judicial e interpretación jurídica. V. Interpretación, deliberación y justificación. VI. Ciencia jurídica y pretensiones de corrección racional. VII. Conclusión. Bibliografía.

\section{Introducción}

El punto de partida del presente trabajo lo constituyen las siguientes tres consideraciones antecedentes. En primer lugar, el hecho de que en el pensamiento de Kelsen no esté desarrollada, propiamente, una teoría de la argumentación jurídica. En segundo lugar, la constatación de que algunos teóricos como Atienza (2003, 2004) consideran explícitamente a la Teoría Pura del Derecho de Kelsen como incompatible con las teorías de la argumentación jurídica y como opuesta a la concepción del derecho como argumentación. En tercer lugar, la exposición de Cuenca (2007) quien, por el contrario, defiende la compatibilidad de la concepción kelseniana del derecho con el constitucionalismo contemporáneo basándose en una interpretación del sistema jurídico como un sistema no puramente dinámico.

Teniendo como marco estas consideraciones, el presente trabajo tiene como objetivo mostrar que dentro de una concepción kelseniana del sistema jurídico la argumentación resultaría ser una actividad imprescindible en el proceso de creación/aplicación del derecho, ya que sería a través de ella como tendrían lugar los distintos procesos de fundamentación, interpretación y justificación de las normas.

Lo anterior, sin embargo, a condición de que se adopte una concepción del sistema jurídico kelseniano que evite caer en alguno de los siguientes extremos. Por un lado, evitando considerar al sistema jurídico como un sistema estático de relaciones puramente lógicas entre las normas. Por otro lado, evitando considerar al sistema jurídico como puramente dinámico, esto es, como un sistema de normas de diversa jerarquía enlazadas exclusivamente por una cadena de mando. 
En un sistema estático no sería necesaria la argumentación, ya que únicamente serían necesarios procesos de inferencia lógica para la determinación del contenido de las normas inferiores a partir del contenido de las superiores. Por su parte, en un sistema puramente dinámico tampoco habría lugar para la argumentación ya que el contenido de las normas inferiores no sería determinado por las superiores, sino que sería decidido arbitrariamente por la autoridad facultada por la norma superior.

Por el contrario, en el presente trabajo se propone considerar al sistema jurídico kelseniano, en un sentido similar a como lo hace Cuenca (2007) como un sistema dinámico en el que las normas de grada superior limitan el contenido posible de las normas de grada inferior, no siendo estas normas, sin embargo, simplemente inferidas sino impuestas por actos de autoridad mediante procesos de toma de decisiones que involucran procesos tanto cognitivos como volitivos.

En lo que corresponde a la función judicial, este enfoque del sistema jurídico mostrará la necesidad y pertinencia de la argumentación en el marco de las actividades de fundamentación de las normas a aplicar, de la determinación de los hechos del caso, en la delimitación del conjunto de interpretaciones posibles, en la deliberación sobre la mejor interpretación adecuada al caso y, por último, en la justificación de la "corrección” moral (ético-política) de la decisión tomada. Con ello, la teoría kelseniana contribuiría a establecer una base para conceptualizar cinco tipos de argumentación que tendrían lugar en el proceso judicial: argumentación fundamentadora, en materia de hechos, interpretativa, deliberativa y justificadora.

Así, lejos de ser incompatible con una concepción del derecho como argumentación, la teoría kelseniana del orden jurídico proporcionaría un marco sólido para conceptualizar el papel de la argumentación jurídica en los distintos momentos del proceso judicial e, incluso, en los distintos contextos de la producción/aplicación del derecho en todos sus niveles. 


\section{Fundamentación y argumentación}

De acuerdo con Kelsen, el derecho es un sistema de normas. Su existencia no es semejante a la de los objetos materiales sino una muy peculiar que se designa con el término "validez". La validez, dice Kelsen, designa la existencia específica de una norma y, por tanto, de un orden normativo (véase Kelsen, 1960/1998: 23 y sigs.). Éste y aquélla "existen" como tales en cuanto que son válidos, de lo que se sigue que una "norma" inválida o "un orden normativo" inválido no son derecho, no son norma u orden normativo, en sentido estricto.

Supongamos que un sujeto A ordena a un sujeto B que prive de su libertad a un sujeto C, diciendo: "Debes encerrar a C". Se trata de un acto de voluntad de A dirigido con intención a B. El sentido de dicho acto es dar una orden a B. Por su parte, el sujeto B se preguntará si la orden que A le ha dado constituye una norma: ¿se trata de algo que $\mathrm{B}$ debe hacer conforme al sistema jurídico, o solamente de algo que A quiere que B haga? Para responder, B debe averigüar si dentro del sistema jurídico existe alguna norma que autorice a A para darle esa orden. Si dicha norma existe, diremos entonces que $\mathrm{A}$ estaba autorizado por la norma $\mathrm{X}$ para ordenar a B que prive de su libertad a C, y que, por lo tanto, la orden de A es, también, una norma jurídica. Si ahora suponemos que en el sistema jurídico no hay ninguna norma que autorice a A para darle esa orden a $\mathrm{B}$, entonces diremos que se trató solamente de un acto de voluntad de A que no "vale" como norma para el sistema jurídico.

Para Kelsen, la determinación de la validez de una norma se lleva a cabo por el criterio de pertenencia de esa norma al orden jurídico existente que, a su vez, remite al hecho de que la norma haya sido producida por un acto de producción regulado por el propio orden jurídico.

La fundamentación de la validez jurídica (existencia) de una norma, por tanto, sólo puede ser establecida por un razonamiento mediante el cual se determine la pertenencia de esa norma al sistema jurídico válido (existente).

Gianformaggio (1994) opina que la fundamentación, entendida de esta manera, no implicaría su discursivización (ni, por lo tanto, la necesidad de una 
actividad argumentativa) ya que no se trataría de un razonamiento que suponga un contexto de interacción comunicativa. La autora señala a este respecto que "más bien, la fundamentación es un esfuerzo intelectual, una tarea en solitario en la que no se requieren compañeros ni oponentes" (Gianformaggio, 1994: 43). Desde esa perspectiva, no habría necesidad de argumentar en el proceso de fundamentación de la validez de una norma.

Por el contrario, en el presente trabajo sostenemos que sí es pertinente considerar a la actividad de fundamentación de la validez de las normas por parte de la autoridad como una actividad dada en el marco de un contexto comunicativo. Ello, debido a que la validez de dichas normas puede ponerse en duda, debiendo la autoridad argumentar sobre las razones en base a las cuales consideró a tal o cual norma como "perteneciente al sistema".

El que la validez de una norma pueda ser puesta en cuestión lo demuestra el hecho de que existan procesos de control de la legalidad y de la constitucionalidad cuyo objeto es, precisamente, determinar si el sentido del acto o los actos de autoridad cuestionados pueden considerarse o no como normas pertenecientes al sistema.

\section{EI sistema jurídico no es puramente dinámico}

Cuenca (2007) ha argumentado a favor de que la fundamentación de la validez de una norma inferior en una superior incluye en Kelsen elementos materiales (o de contenido) y no puramente formales, a condición de que hagamos una lectura más atenta de los textos del austriaco. Ello cuestionaría la interpretación corriente del sistema jurídico kelseniano como un sistema dinámico puro al incorporar un “elemento estático" en la determinación de la validez.

Cuenca (2007) argumenta, en primer lugar, que el sistema jurídico kelseniano no está compuesto por normas de pura delegación, sino también por normas sustantivas que disciplinan el contenido de las normas inferiores, como se ve claramente en la caracterización que hace Kelsen de la "grada" 
correspondiente a la legislación general, donde el austriaco afirma la unidad del derecho sustantivo y el adjetivo.

En segundo lugar, la autora explica que la tesis kelseniana según la cual la validez depende de la forma de creación implica que dicha creación debe ocurrir también conforme al contenido de las normas superiores ya que "los contenidos relevantes para la validez son requisitos materiales formalmente determinados" $\mathrm{y}$ no puramente formales (Cuenca, 2007: 16).

En tercer lugar, la autora argumenta que un sistema puramente dinámico admitiría incoherencias en el contenido de las normas, pero que no es el caso de Kelsen quien no admite la validez simultánea de normas contradictorias. Por último, en cuarto lugar, señala que la teoría de la interpretación de Kelsen implica la obligación por parte de la autoridad de grada inferior de sujetarse al contenido de la norma de grada superior.

$\mathrm{Al}$ respecto de todo lo anterior conviene recordar, por nuestra parte, que el propio Kelsen es explícito en afirmar que desde la misma Constitución general es posible establecer una delimitación del contenido de las normas de todo el sistema jurídico. Dice el austriaco a este respecto: "La constitución que regula la producción de normas generales puede determinar también el contenido de las futuras leyes, y las constituciones positivas lo hacen no poco frecuentemente, al prescribir, o excluir ciertos contenidos" (Kelsen, 1960/1998: 234).

Además, como veremos, tanto en la caracterización que hace Kelsen de la función judicial como en su teoría de la interpretación, el austriaco señala que la decisión de la autoridad jurisdiccional está determinada por la norma general no sólo en cuanto a su forma de producción sino, también, en cuanto a su contenido.

De modo que, de acuerdo con todo lo anterior, la validez de una norma estará determinada no sólo por la circunstancia de que su acto de producción esté autorizado por una norma superior (y, en última instancia, por la Constitución) sino también por la condición de que su contenido corresponda con el delimitado por dichas normas superiores (y, en última instancia, por la Constitución). 
Con respecto a esto es importante hacer notar, en el caso del sistema jurídico mexicano, que lo anterior implicaría la necesidad de que el contenido de todas las normas producidas dentro de dicho sistema sea compatible con el bloque de constitucionalidad definido a partir de las reformas de 2011.

Por todo lo anterior, es posible concluir que la presencia de este elemento de contenido en la fundamentación kelseniana de la validez haría a la concepción kelseniana compatible con el pensamiento constitucionalista contemporáneo en la medida en que permite dar cuenta de la proyección dinámica de los elementos sustantivos y adjetivos de la Constitución sobre el conjunto del sistema jurídico.

\section{Función judicial e interpretación jurídica}

El análisis de la función judicial y de la interpretación jurídica hay que contextualizarlo en el marco de la concepción kelseniana de la estructura escalonada del orden jurídico pero desde una perspectiva dinámica, esto es, considerando al sistema jurídico en movimiento.

Hay que ver a la función judicial y a la interpretación como procesos presentes en la creación/aplicación del derecho "en su tránsito de una grada superior a una inferior" (Kelsen, 1960/ 1998: 349). Como dice Kelsen: "la sentencia judicial es la continuación, no el comienzo, del proceso de producción de derecho" (Kelsen, 1960/ 1998: 263).

El derecho en cuanto sistema dinámico que regula su propia producción va adquiriendo un mayor grado de concreción en su determinación sucesiva que va de la Constitución a la Ley general, de ésta a la norma individualizada y, finalmente, al acto de ejecución, constituyendo todo ello un solo proceso que culmina, precisamente, en dicho acto de ejecución.

En cada "descenso" de una grada hacia otra tiene lugar un proceso de interpretación de la norma superior que permite a la autoridad producir la norma inferior dentro del marco determinado por dicha norma superior y, en última instancia, por la Constitución. Dentro de todo este proceso, por tanto, la función 
judicial constituye solamente una etapa intermedia. Dice Kelsen:

Desde el punto de vista de una consideración orientada a la dinámica del derecho, la implantación de una norma individual por el tribunal constituye una etapa intermedia en el proceso iniciado con la erección de la constitución, y que, pasando por la legislación y la costumbre, lleva a la sentencia judicial y de ésta, a la ejecución de la sanción. Este proceso, en el cual el derecho se autorreproduce permanentemente, va de lo general (o abstracto) a lo individual (o concreto). Se trata de un proceso de una individualización o concretización en incremento ininterrumpido (Kelsen, 1960/1998: 246).

La aplicación del derecho que lleva a cabo el órgano judicial, entonces, ha de verse como un proceso de individualización de la norma general. Según Kelsen, para tal individualización el órgano jurisdiccional tiene que establecer si en el caso que se le presenta se dan en concreto las condiciones que la norma general determina en abstracto para la aplicación. Dichas condiciones son de dos tipos. En primer lugar, la determinación de la norma a aplicar y, en segundo lugar, la determinación del hecho condicionante de la sanción.

En la determinación de la norma a aplicar el órgano jurisdiccional lleva a cabo la actividad de fundamentación que ya hemos descrito. Kelsen caracteriza a dicha actividad como una función constitutiva ya que la autoridad debe establecer la validez de la mencionada norma. La norma solo se hace aplicable al caso mediante la comprobación de su validez que hace la autoridad jurisdiccional. En concreto, la autoridad debe determinar si la norma a aplicar ha sido producida conforme a las demás del sistema y, en particular, conforme a la Constitución.

Por otro lado, en la determinación del hecho condicionante la sentencia judicial cumple también una función constitutiva en el sentido de que tal hecho debe ser constituido (producido) procesalmente: no es el hecho en sí el que será condición de la sanción sino el hecho procesalmente establecido. Este momento, presente en la actividad jurisdiccional, abre evidentemente el espacio para la argumentación en materia de hechos la cual, según Atienza $(2001,2003)$ es uno de los aspectos más descuidados actualmente por la teoría estándar de la argumentación. 
Ahora bien, además de establecer la validez de la norma por aplicar y el hecho condicionante, el órgano jurídico deberá también interpretar la norma a aplicar. Dice Kelsen que cuando se habla de interpretación el caso en el que más se piensa es en la interpretación de la ley en su aplicación al hecho concreto a través de la sentencia judicial o de una resolución administrativa. No obstante, en este punto es importante considerar que la interpretación, de acuerdo con Kelsen, es un proceso que está presente en todos momentos de la creación/aplicación del derecho ya que tenemos también una interpretación de la Constitución, una interpretación de los tratados internacionales, una interpretación de las normas individualizadas por la autoridad ejecutora, etcétera. En suma, dice el austriaco, tenemos "una interpretación de todas las normas jurídicas, en tanto deben recibir aplicación" (Kelsen, 1960/1998: 349).

Ello significa que la interpretación es un proceso siempre presente y no ceñido únicamente a determinados casos (e.g. a "casos difíciles" por la autoridad judicial) sino que toda norma debe recibir interpretación en tanto debe recibir aplicación; es decir, "que la interpretación es necesaria para todo acto de aplicación del derecho" (Troper, 1994: 82).

La interpretación, dice Kelsen, "es un procedimiento espiritual que acompaña al proceso de aplicación del derecho, en su tránsito de una grada superior a una inferior" (Kelsen, 1960/1998: 349). La relación entre una grada superior y una inferior, dice el austriaco, es una relación de determinación o de obligación en el sentido de que la norma de grada superior determina: a) el órgano que la aplicará, b) el procedimiento que seguirá en su aplicación y c) el contenido de la norma jurídica que será producida.

Sin embargo, la determinación de la norma de grada inferior por la de grada superior nunca es completa ya que la norma superior no puede determinar en todos los sentidos el acto mediante el cual se la aplicará. Dice Kelsen:

Hasta la orden más minuciosa tiene que dejar al ejecutor una cantidad de determinaciones. Si el órgano A dispone que el órgano B ponga en prisión al súbdito $\mathrm{C}$, el órgano $\mathrm{B}$ tendrá que resolver a su criterio cuándo y donde y cómo se pondrá en efecto la orden de prisión, 
decisiones que dependen de circunstancias externas que el órgano que dio la orden no previó y que, en buena parte, tampoco pudo prever (Kelsen, 1960/1998: 350).

De ello se sigue que todo acto de aplicación/producción de derecho sólo está determinado parcialmente por el derecho quedando en parte indeterminado. Tal indeterminación puede referirse tanto al hecho condicionante, como a la consecuencia condicionada, y puede haber sido establecida intencional o no intencionalmente por el órgano que la produjo.

La indeterminación intencional tiene lugar en la promulgación de una norma meramente general la cual se efectúa siempre bajo el supuesto de que la norma individual que surgirá en su aplicación "continuará el proceso de determinación que configura el sentido mismo de la secuencia graduada de normas jurídicas"; la indeterminación no intencional, en cambio, "puede ser la consecuencia no buscada de la forma de ser de la norma jurídica que debe ser aplicada mediante el acto en cuestión" (Kelsen, 1960/1998: 350).

Debido a esta indeterminación es que se ofrecen para la aplicación de la norma general varias posibilidades interpretativas. Como todas esas posibilidades son jurídicamente equivalentes, la elección de cualquiera de ellas estará fundada en derecho. Así, Kelsen habla del derecho aplicable como "un marco dentro del cual hay varias posibilidades de aplicación" (Kelsen, 1960/ 1998: 351). Dice Kelsen: "todo acto es conforme a derecho si se mantiene dentro de ese marco, colmándolo en algún sentido posible” (Kelsen, 1960/ 1998: 351) y, también:

Que una sentencia judicial está fundada en ley no significa, en verdad, sino que se mantiene dentro del marco que le ley despliega; sino que es una de las normas individuales -y no la norma individual- que pueden ser producidas dentro del marco ofrecido por la norma general (Kelsen, 1960/1998: 351-352)

Aquí hay que notar dos cosas importantes. En primer lugar, el hecho de que una resolución esté dentro del marco señalado significa que la norma individualizada está fundamentada en la norma general y en la Constitución y que, por tanto, tiene validez. En segundo lugar, ello no tiene nada que ver con su 
corrección o incorrección moral sobre la cual (todavía) no se ha dicho nada, ya que "Kelsen nunca confundió 'validez' con ‘corrección”” (Gianformaggio, 1994: 42).

En suma, dado que toda norma es sujeta de interpretación en la medida en que es necesario darle aplicación, y toda vez que siempre existirán múltiples alternativas interpretativas, la producción de una nueva norma será válida en la medida en que se encuentre dentro de ese abanico de alternativas interpretativas. Como se explicará en los siguientes apartados, la determinación del marco de interpretaciones será, para Kelsen, una labor científica; en cambio, la elección de alguna de dichas interpretaciones como "correcta" será, para el austríaco, una labor política.

\section{Interpretación, deliberación y justificación}

De acuerdo con Kelsen, en la interpretación llevada a cabo por un órgano de aplicación del derecho cabe distinguir conceptualmente dos momentos: a) un proceso de interpretación cognoscitiva cuyo resultado es la determinación del marco de posibilidades de aplicación de la norma general, y b) una elección entre esas posibilidades. Dice Kelsen:

Si hubiera que caracterizar no sólo la interpretación de la ley por parte de los tribunales u órganos administrativos, sino en forma enteramente general, la interpretación del derecho por los órganos de aplicación del derecho, habría que decir: en la aplicación del derecho por un órgano jurídico, la interpretación cognoscitiva del derecho aplicable se enlaza con un acto de voluntad en el cual el órgano de aplicación de derecho efectúa una elección entre las posibilidades que la interpretación cognoscitiva muestra. Con ese acto o bien se produce una norma jurídica de nivel inferior, o se lleva a cabo el acto coactivo estatuido por la norma jurídica aplicable (Kelsen, 1960/ 1998: 354).

Como se puede apreciar claramente en el pasaje citado, el segundo de los momentos de la interpretación llevada a cabo por la autoridad, esto es, la elección, no es otra cosa sino el mismo acto de instauración de la norma jurídica por él 
creada (i.e., es nuestro caso, la resolución judicial). En la medida en que dicho acto de instauración es una decisión, Kelsen lo caracteriza como una función volitiva, la cual en nuestro caso se referiría al acto de creación de la resolución judicial: "el alcanzar una norma individual a través del proceso de aplicación de la ley, es, en tanto se cumple dentro del marco de la norma general, una función volitiva" (Kelsen, 1960/1998: 353-354).

Ahora bien, considerando el primer momento del proceso de la interpretación auténtica, habría que recalcar que Kelsen caracteriza a este momento como una actividad cognoscitiva, es decir, como un conocimiento del derecho por aplicar. El derecho por aplicar, recordemos, sólo constituye un marco dentro del cual están dadas varias posibilidades de aplicación, según había afirmado el austriaco. Por tanto, se trata simplemente de llegar al conocimiento de ese conjunto de posibilidades dadas. De lo anterior deriva la posibilidad de determinar un único "marco de aplicaciones posibles" ya que, tratándose de una actividad de conocimiento podrá determinarse la verdad o falsedad de sus resultados. Por ejemplo, deberá ser posible juzgar si una determinada "interpretación" cae, efectivamente, dentro del marco de interpretaciones posibles o no. Así, en la medida en que esta calificación pueda ser problemática dará lugar a un proceso de argumentación interpretativa cuya finalidad será exponer buenas razones para decidir si una determinada opción interpretativa forma, realmente, parte del marco de posibilidades de aplicación o si, por lo contrario, cae fuera de él.

Por otro lado, según Kelsen, la actividad de conocimiento del derecho aplicable al caso no puede ir más allá de la determinación de ese marco de posibilidades de aplicación ya que no existe, desde un punto de vista orientado hacia el conocimiento del derecho positivo, "criterio alguno con cuyo fundamento pueda preferirse una posibilidad dada dentro del marco" (Kelsen, 1960/1998: 352). Por lo tanto, no es posible llegar a través de este conocimiento a la determinación de una única interpretación "correcta", ya que cognoscitivamente, todas las posibilidades dadas tienen el mismo valor. Dice el austriaco:

Por lo tanto, la interpretación de una ley no conduce necesariamente a una decisión única, 
como si se tratara de la única correcta, sino posiblemente a varias, todas las cuales -en tanto son cotejadas solamente con la ley que haya de aplicarse- tienen el mismo valor, aunque solo una de ellas se convertirá en derecho positivo en el acto del órgano de aplicación del derecho, en especial, en el acto del tribunal (Kelsen, 1960/1998: 351-352).

Así, pues, desde un punto de vista estrictamente jurídico, todas las posibilidades de aplicación dadas dentro del marco son equivalentes. La autoridad producirá una resolución válida "conforme a derecho" eligiendo cualquiera de ellas. Esta elección, por tanto, no puede ser determinada por el conocimiento jurídico, sino solo el marco dentro del cual ella ha de tener lugar.

El problema que se le plantea a la autoridad, por tanto, es la cuestión de qué decidirá y de cómo tomará esa decisión dentro del marco de posibilidades de aplicación, en el entendido de que, a partir de este momento, el conocimiento jurídico no le brindará ningún criterio para juzgar de la "corrección” de la elección que tome.

Ello es así, diría Kelsen, porque en este momento ya no se trata de conocer lo que la norma general dispone sino de decidir (aunque dentro del derecho) cómo el derecho debe ser. No se trata, por tanto, de un asunto del conocimiento del derecho sino de una cuestión de política jurídica:

La pregunta de cuál sea la posibilidad "correcta", en el marco del derecho aplicable, no es -según los supuestos previos- ninguna pregunta dirigida al conocimiento del derecho positivo, no es una pregunta teórica-jurídica, sino que es un problema político (Kelsen, 1960/1998: 353).

Así pues, el problema que se le plantea a la autoridad es la cuestión de qué decidirá y de cómo tomará esa decisión dentro del "marco de posibilidades de aplicación". Se trata aquí, por tanto, de un proceso deliberativo.

Vallet de Goytisolo (2009) considera que la deliberación debe diferenciarse de la argumentación ya que esta última supone un contexto comunicativo mientras que la primera refiere a un proceso mental que ocurre en solitario. Sin embargo, en el presente trabajo consideramos que la existencia de 
la argumentación de tipo deliberativo es evidente, actualmente, por lo menos en la actividad desarrollada por órganos colegiados. Por ejemplo, en el Pleno de la Suprema Corte de Justicia de la Nación, los Ministros suelen discutir los pros y contras de las distintas opciones interpretativas, empleando para ello argumentos acerca de las diversas posiciones antes de tomar una decisión.

Para comprender mejor la argumentación deliberativa conviene desde ahora diferenciarla respecto de la argumentación justificatoria. Para ello nos apoyaremos en dos criterios. El primero es tomado de la teoría de toma de decisiones; el segundo, de la teoría de la argumentación.

En la teoría de toma de decisiones suele distinguirse entre una fase predecisional y una postdecisional. En ese marco, la deliberación es caracterizada como un momento predecisional el cual comienza "cuando la persona se da cuenta de la necesidad de tomar una decisión” (Mann, 2008). En cambio, la justificación corresponde a un momento post decisional, en el cual se utilizan razonamientos para establecer la "corrección" de la decisión ya tomada.

La teoría estándar de la argumentación, por su parte, distingue entre contexto de descubrimiento y contexto de justificación. Mientras que el contexto de descubrimiento hace referencia al proceso mediante el cual la autoridd llega a tomar una decisión, el contexto de justificación se refiere a la manera en que dicha autoridad justifica la decisión tomada. Conforme a ello se distinguen razones explicativas y razones justificatorias. Las primeras hacen referencia a los móviles psicológicos, el contexto social, las circunstancias ideológicas, etc. que determinan al juez a dictar una resolución. Las razones justificatorias, en cambio, se refieren a las razones que el órgano en cuestión aduce para mostrar que su decisión es correcta, aceptable o justificada (Atienza, 2001, 2003).

Tenemos, entonces, aquí otros dos contextos en los que puede tener lugar la argumentación jurídica en el marco del funcionamiento del sistema jurídico kelseniano: la deliberación y la justificación. La primera tendría como finalidad comparar a las distintas opciones disponibles con el fin inclinarse por alguna; la segunda tendría como finalidad exponer buenas razones a favor de que la resolución tomada "es correcta". 
Siendo la deliberación parte del contexto de descubrimiento y existiendo allí únicamente razones explicativas de la decisión, la argumentación podría no estar necesariamente implicada en el proceso deliberativo. De hecho, tanto la teoría de toma de decisiones en general (Mann, 2008) como algunos estudios empíricos realizados sobre toma de decisiones judiciales en particular (Arce, Fariña y Seijo, 2005; Fariña, Arce y Novo, 2002), más bien parecen indicar la relativa ausencia de argumentación durante la fase predecisional, pues han mostrado la presencia de niveles bajos de actividad cognitiva desplegada durante estos procesos afectando a la calidad de las resoluciones, ya que implica la presencia de sesgos cognitivos. Sin embargo, en la medida en que estos fenómenos son conceptualizados psicológicamente como estrategias de ahorro cognitivo, la presencia de una actividad argumentativa durante la deliberación podría tener un efecto favorable en la calidad de las decisiones.

Ahora bien, dicha actividad argumentativa (argumentación deliberativa) conceptualmente podría tener por objeto que la autoridad compare las diversas alternativas dadas dentro del "marco de posibilidades de aplicación" inclinándose hacia alguna de ellas. Evidentemente, dichas inclinaciones podrían ocurrir en principio debidas fundamentalmente a factores subjetivos; sin embargo, en la medida en que el contexto comunicativo que supone la argumentación implica exigencias de racionalidad, se plantea el problema de tener criterios que determinen la "corrección" de la decisión a tomar. Ello no implica entrar en el contexto de justificación sino anticipar las razones que bien podrían esgrimirse en dicho contexto.

Ahora bien, ¿de qué criterios de corrección estaríamos hablando aquí? En la medida en que Kelsen distingue entre "validez" y "corrección”, y en la medida en que aquélla se determina por el conocimiento jurídico, más no ésta, los criterios o patrones para juzgar de la "corrección" de una determinada decisión tendrían qué ser, necesariamente, metajurídicos y, en particular, de naturaleza ético-política.

A este respecto conviene enfatizar que Kelsen admite esta posibilidad de que el derecho pueda ser calificado de "justo" o "injusto", de "correcto" o 
DERECHO GLOBAL. ESTUDIOS SOBRE DERECHO Y JUSTICIA

"incorrecto". Solamente aclara que dicha calificación no proviene de criterios jurídicos sino ético-políticos. De hecho, señala el austriaco, es como si el derecho "delegara" en otros sistemas normativos el establecimiento del acto a realizar. Cito in extenso:

En tanto en la aplicación de la ley, por añadidura de la determinación necesaria del marco dentro del cual debe cumplirse el acto que se efectuará, puede tener lugar una actividad cognoscitiva del órgano de aplicación, no se trataría de un conocimiento del derecho positivo, sino de otras normas que pueden desembocar aquí en el proceso de producción de derecho: normas morales, normas de justicia, juicios de valor sociales, etcétera, que se suele denominar con rótulos tales como: "bien común", "interés del Estado", "progreso", etcétera. Desde el punto de vista del derecho positivo nada cabe decir sobre su validez y verificabilidad. Desde ese punto de vista, tales especies de determinación sólo pueden ser caracterizadas negativamente: son determinaciones que no provienen del derecho positivo mismo. En relación con ellas, la realización del acto jurídico dentro del marco de la norma jurídica aplicable es libre, es decir, librado a la libre discrecionalidad del órgano llamado a efectuar el acto, como si el derecho positivo mismo delegara en ciertas normas metajurídicas, como la moral, la justicia, etcétera; pero de ese modo esas normas se transformarían en normas jurídicas positivas (Kelsen, 1960/1998: 353-354).

Vemos entonces que la tarea de justificación que puede tener lugar tanto en el ámbito de la argumentación deliberativa (contexto de descubrimiento) como en de la argumentación justificatoria (contexto de justificación) está guiada, para Kelsen, por criterios de corrección, aunque estos criterios serían no científicos sino metajurídicos y, en particular, ético-políticos.

\section{Ciencia jurídica y pretensiones de corrección racional}

Una de las principales preocupaciones de las teorías de la argumentación contemporáneas está en la determinación de posibles criterios de corrección para las decisiones judiciales. Se entiende, de principio, que esta preocupación es, fundamentalmente, de naturaleza ético-política: se trata de determinar criterios de justificación (de racionalidad práctica) que permitan un control racional 
de la actividad jurisdiccional en el contexto del fortalecimiento del Estado democrático. Según Atienza (2004) uno de los factores que explican el actual auge de las teorías de la argumentación jurídica es precisamente el hecho de que se ha venido operando un cambio general en los sistema jurídicos en el paso del Estado legislativo al Estado constitucional ya que el avance del Estado constitucional, dice, va acompañado de un incremento de la tarea justificativa de los órganos públicos $\mathrm{y}$, por tanto, de una mayor demanda de argumentación, lo que permitiría alcanzar paulatinamente el ideal del sometimiento del poder a la razón. Dice Atienza: "En realidad, el ideal del Estado constitucional (la culminación del Estado de Derecho) supone el sometimiento completo del poder al Derecho, a la razón: la fuerza de la razón, frente a la razón de la fuerza” (Atienza, 2004: 75).

Desde una perspectiva como ésta es comprensible a primera vista que existan reservas frente a una concepción que, como la kelseniana, propone: a) una ciencia del derecho "pura" que se limite a describir al derecho existente sin pretender formular afirmaciones acerca de su corrección o incorrección; b) un pensamiento ético-político que adopta explícitamente una postura de relativismo moral. Veremos, sin embargo, que son estos dos puntos, precisamente, los que en la perspectiva kelseniana permiten aspirar a un sometimiento del poder a la razón.

Como hemos visto, para Kelsen siempre existen diversas posibilidades de interpretación de la norma los cuales configuran un "marco" de interpretaciones posibles. Dentro de tal marco la autoridad deberá elegir cuál aplicar. La función del científico-jurista consistirá en mostrar de la manera más objetiva posible dicho marco de posibilidades interpretativas evitando, en todo momento, que sus inclinaciones o preferencias subjetivas le lleven a intentar defender a alguna de dichas posibilidades de interpretación como "La (científicamente) correcta".

Evidentemente el jurista, como todo ciudadano, puede inclinarse por tal o cual interpretación. Sin embargo, es importante aclarar que dicha inclinación la tendrá ese sujeto en tanto que ciudadano, no en cuanto que jurista. El jurista, en cuanto científico del derecho, debe limitar su tarea a la descripción objetiva (i.e., lo más objetiva posible) del "marco de interpretaciones". 
Al exponer el marco de posibilidades interpretativas absteniéndose de defender una determinada interpretación como "La Correcta" en nombre de La Ciencia o de La Razón el jurista contribuye al diálogo democrático en un triple sentido:

a) Al mostrar que todas esas posibilidades de interpretación tienen el mismo valor científio, el científico-jurista kelseniano hace evidente que las preferencias que podamos tener sobre tales o cuales interpretaciones no derivan de criterios científicos sino de disposiciones subjetivas tales como creencias o convicciones morales, religiosas o políticas, lo que forzará a dialogar a todo aquél que quiera hacer prevalecer su elección sobre las de los demás;

b) Especialmente, el jurista hará evidente que la elección que haga la autoridad de tal o cual interpretación será una elección ético-política entre varias posibles y que, ante la ciudadanía, por tanto, la autoridad deberá justificar dicha elección en esos términos ético-políticos; y,

c) En tercer término, estará evitando que se defiendan convicciones políticas, éticas, religiosas, etc., en nombre de la ciencia sustrayendo a ésta, a la ciencia, del control político, y contribuyendo con ello a la realización del ideal de una ciencia libre que, liberada de su uso ideológico, pueda contribuir eficientemente al progreso de la técnica social (i.e. a formas más eficientes de organizar la convivencia humana).

Como consecuencia de todo lo anterior, si el jurista kelseniano se limitara a señalar las interpretaciones posibles de la norma sin señalar la corrección de alguna de ellas, esa labor no resultará ni sencilla ni inútil como algunos la han juzgado (véase por ejemplo, Cossio, 1964; Errázuriz, 1987; Atienza, 2004). Al contrario, como vemos, es precisamente el valor de la objetividad científica el que permite exhibir la naturaleza política del acto jurisdiccional y, por tanto, el que desencadena la necesidad de argumentar para justificar ético-políticamente las decisiones que, así, ya no pueden cobijarse bajo el disfraz de la ciencia para ganar legitimidad.

La ciencia, como conocimiento, dice Kelsen, tiene la tendencia inmanente 
a descubrir su objeto; la ideología, en cambio, tiende a encubrir la realidad (Kelsen, 1960/1998: 122). Por ello, hay en Kelsen un principio que determina la racionalidad de la argumentación democrática y que podemos enunciar de la siguiente manera: no defender convicciones políticas o morales en nombre de la ciencia como si se tratara de principios absolutos e universales.

Ahora bien, aquí es donde entra el presupuesto relativista de Kelsen. Para comenzar debemos señalar que el relativismo moral es, para Kelsen, la condición de posibilidad del diálogo democrático (Araos, 2008; Kelsen, 1933/2003). En efecto, como primer aspecto del relativismo kelseniano podemos señalar que éste implica reconocer, en principio, que el conocimiento de El Bien Absoluto está más allá de los límites del conocimiento humano; que, con relación al desconocimiento de E1 Bien, los seres humanos estamos en condiciones de igualdad. El reconocimiento de la igualdad es correlativo de un sentido de fraternidad (de no vivirse como "superior a los demás", perspectiva típica del autócrata), de una tendencia a la autocrítica y de un sentimiento de responsabilidad; por lo tanto, de una actitud de apertura a dialogar, a escuchar al otro (Kelsen, 1933/2003).

Un segundo aspecto del principio relativista es que éste, para Kelsen, no implica indiferencia moral ya que, según afirma el austriaco:

Una doctrina relativista de los valores no significa -como múltiples veces ha sido mal entendido- que no haya valores y, en especial, que no exista justicia alguna, sino que no hay valores absolutos, sino solo relativos; que no hay una justicia absoluta, sino relativa (Kelsen, 1960/1998: 80).

Evidentemente una moral relativa no puede cumplir la función, dice Kelsen, de proveer de un patrón absoluto para la evaluación de un determinado orden jurídico-positivo; pero un patrón semejante, afirma "no cabe encontrarlo en el camino del conocimiento científico" (Kelsen, 1960/1998: 80). Ello no significa, sin embargo, que no exista patrón alguno. Dice el austriaco:

Cada sistema moral puede servir como patrón semejante. Pero es necesario tener conciencia, cuando se juzga la conformación de un orden jurídico positivo como "moral”, como bueno o 
malo, justo o injusto, que el patrón es relativo, que no está excluida otra evaluación a partir de otro sistema moral; que cuando se considera un orden jurídico como injusto, conforme al patrón de un sistema moral, el mismo orden jurídico puede ser estimado justo, ateniéndose al patrón de otro sistema moral (Kelsen, 1960/1998: 80).

Por tanto, se puede argumentar a favor de la corrección o incorrección moral de tal o cual acto, norma o, en nuestro caso, de tal o cual decisión judicial, desde la perspectiva de determinado sistema moral. Significa, entonces, que sí es posible defender determinados criterios de corrección. Lo único que se pide aquí (como actitud democrática, basada en la conciencia de no poseer el conocimiento científico de El Bien) es no defender al propio sistema moral como si fuera un patrón absoluto, sino en la conciencia de que pueden existir otros criterios de corrección.

Ahora bien, si esto es así, si de lo que se trata es de argumentar a favor de una determinada pretensión de corrección pero admitiendo, al mismo tiempo, la existencia de otros patrones diferentes se podría preguntar ¿qué sentido tiene, entonces, la argumentación? ¿para qué se argumenta si, finalmente, nunca se llega a un patrón absoluto, a una solución definitiva, a una única decisión correcta? La respuesta de Kelsen es muy sencilla: se argumenta para "atraer adeptos en una libre competencia por las mentes de los hombres" (Kelsen, 1933/ 2003: 261); es decir, se argumenta para lograr el asentimiento de los otros, buscando que se adhieran a nuestra justificación. Incluso podríamos decir que se argumenta con la pretensión de que los otros reconozcan como razonable nuestra justificación aunque no se adhieran, necesariamente, a nuestra postura ya que serían posibles, también, otras posturas razonables.

Por último, si de lo que se trata es de buscar ese asentimiento o adhesión de los otros a través de la actividad argumentativa, bajo el presupuesto de que nadie tiene el conocimiento de El Bien Absoluto, entonces actúa en contra de esta libre discusión, vulnera el diálogo democrático, aquél que pretende defender su patrón valorativo como si se tratara de una Verdad de la ciencia, de un valor absoluto. 
Por tanto, la condición bajo la cual es posible el diálogo democrático, la condición de la racionalidad de esta libre discusión no es otra que el principio antes mencionado el cual no solo protege a la libre discusión sino también, a la objetividad científica: no defender convicciones políticas o morales, en nombre de la ciencia, como si se tratara de principios absolutos e universales.

Así, respecto de la función judicial, esta concepción kelseniana implicaría que el juez tendrá que argumentar a favor de la razonabilidad de sus criterios de corrección frente a la ciudadanía, debido a que su decisión no podrá ser considerada "científica" o "verdadera", sino sólo defendible por argumentos que logren ganar la adhesión de los ciudadanos en el marco de otros discursos en competencia por ganar dicha adhesión. Así, este principio de razonabilidad kelseniano (que combina el relativismo moral con una fe en la objetividad de la ciencia) constituiría la base de la libre discusión democrática al despojar a las decisiones judiciales de pretensiones de absolutismo y cientificidad.

\section{Conclusión}

Del acercamiento al análisis kelseniano hemos encontrado algunos contextos en los que, presumiblemente, tiene o podría tener lugar la argumentación en la toma de decisiones judiciales. En concreto, podría hablarse de cinco tipos de argumentación: a) argumentación fundamentadora, cuyo objetivo sería establecer la validez de la norma a interpretar y a aplicar; b) argumentación en materia de hechos, para determinar los hechos condicionantes de la aplicación; c) argumentación interpretativa, para delimitar el marco de interpretaciones posibles; d) argumentación deliberativa, para ponderar las opciones interpretativas en la búsqueda de la mejor alternativa de acuerdo con criterios ético-políticos; y, e) argumentación justificatoria, para justificar la corrección de la decisión tomada, de acuerdo con criterios ético-políticos.

Según hemos visto, las argumentaciones fundamentadora y en materia de hechos son establecidas por el propio Kelsen como actividades que tienen lugar en el seno del proceso judicial. La argumentación interpretativa no es 
postulada explícitamente por Kelsen pero deriva de una necesidad de su propia teoría. Por su parte, las argumentaciones deliberativa y justificatoria se imponen como una actividad deseable en el contexto de descubrimiento y de justificación, respectivamente. Aunque las argumentaciones deliberativa y justificatoria no están necesariamente implicadas por la Teoría Pura tampoco están negadas; no obstante, su racionalidad dependerá de un principio democrático kelseniano que combina la creencia en la objetividad de la ciencia con el relativismo moral.

Por tanto, el pensamiento kelseniano no solo es compatible con una concepción del derecho como argumentación sino que, de hecho, nos brinda un marco para conceptualizar sistemáticamente diversos contextos donde la argumentación puede tener o tiene lugar, en el proceso de producción/aplicación del derecho. Además, su relativismo moral permite sentar las bases para una concepción de la argumentación que busque criterios de corrección surgidos del libre diálogo democrático y no impuestos por un supuesto conocimiento, como dice Kelsen, de El Bien Absoluto.

\section{Bibliografía}

Araos, J. (2008). Relativismo, tolerancia y democracia en H. Kelsen. Veritas, 3(19), 253-269. Recuperado el 23 de febrero de 2018 desde http://www.redalyc.org/articulo.oa?id=291122940003

Arce, R., Fariña, F., y Seijo, D. (2005). Razonamientos judiciales en procesos de separación. Psicothema, 17(1), 57-63. Recuperado el 23 de febrero de 2018 desde http://www.psicothema.com/pdf/3064.pdf Atienza, M. (2001). Cuestiones judiciales. México: Fontamara. Atienza, M. (2003). Las razones del derecho. Teorías de la argumentación jurídica. México: Universidad Nacional Autónoma de México. Atienza, M. (2004). El derecho como argumentación. México: Fontamara. Cossio, C. (1964). La teoría egológica del derecho y el concepto jurídico de libertad (2a. ed.). Buenos Aires: Abeledo-Perot.

Cuenca, P. (2007). Acerca del carácter dinámico del sistema jurídico en 
la teoría de Kelsen y su compatibilidad con el constitucionalismo. Cuadernos Electrónicos de Filosofía del Derecho, 15. Recuperado el 23 de febrero de 2018 desde https:/www.uv.es/CEFD/15/cuenca.pdf Errázuriz, C. (1987). Introducción crítica a la doctrina jurídica de Kelsen. Santiago de Chile: Editorial Jurídica de Chile.

Fariña, F., Arce, R. y Novo, M. (2002). Heurístico de anclaje en las decisiones judiciales. Psicothema, 14(1), 39-46. Recupeado el 23 de febrero de 2018 desde http://www.psicothema.com/pdf/684.pdf Gianformaggio, L. (1994). Estudios sobre Kelsen (Trads., P. Larrañaga y M. Serrano). México: Fontamara.

Kelsen, H. (1994). Teoría general de las normas (Tr. H. Delory). México: Trillas. (Obra original publicada en 1979).

Kelsen, H. (2003). Forma de Estado y visión del mundo. En O. Correas (Ed.),Elotro Kelsen (pp. 223-241). México: Centro de Investigaciones Interdisciplinarias en Ciencias y Humanidades UNAM Coyoacan. (Trabajo original publicado en 1933).

Kelsen, H. (1998). Teoría pura del derecho (Tr., R. Vernengo). México: Porrúa. (La obra original corresponde a la 2a. edición alemana de 1960).

Mann, L. (2008). Elementos de psicología social (Tr., M. Llanos). México: Limusa.

Troper, M. (1994). Pour une théorie juridique de l'etat. París: Presses Universitaires de France.

Vallet de Goytisolo, J.B.(2009). El razonamientojudicial. Anales de la Real Academia de Jurisprudencia y Legislación, 39, 15-28. Recuperado el 20 de mayo de 2018 desde http://fundacioneliasdetejada.org/wpcontent/uploads/2014/03/ANA15-015-028.pdf 
